\title{
Preventing, Delaying, or Masking Type 2 Diabetes With Metformin in the Diabetes Prevention Program?
}

\author{
ANDRE J. SCHEEN, MD, PHD \\ From the Division of Diabetes, Nutrition and Metabolic Disorders, Department of Medicine, CHU Sart Tilman, Liège, Belgium.
}

The secondary analysis of the Diabetes Prevention Program (DPP) showed that withdrawal of metformin for 1-2 weeks resulted in a trend to a higher conversion rate from impaired glucose tolerance to diabetes as compared with the placebo group (1). This resulted in a reduction by $26 \%$ of the so-called prevention effect of metformin, i.e., from $31 \%$ in the primary analysis (2) to $25 \%$ in the secondary analysis, a reduction in the incidence of diabetes that, however, remained highly significant as compared with placebo. The same trend was observed in the Study to Prevent (STOP)-NIDDM trial with acarbose after a longer washout period of 3 months (3). As previously discussed (4) and emphasized in a previous Diabetes Care Editorial (5), one key question is to know whether the positive results with metformin in the DPP (2) or with acarbose in the STOP-NIDDM trial (3) could be interpreted as a real prevention of the disease or only as a delay in its progression, or even simply as a masking effect due to the metabolic effect of the drug. It was astonishing that a possible direct effect of metformin was not discussed in the original paper of the DPP, despite the fact that the results were initially presented without any washout period (2). Indeed, a significant improvement of insulin sensitivity and glucose metabolism was consistently demonstrated in placebo-controlled randomized clinical trials after only $48 \mathrm{~h}$ treatment with metformin ( $850 \mathrm{mg}$ twice daily), both in insulin-resistant normoglycemic first-degree relatives of diabetic patients during a euglycemic insulin clamp (6) and in hyperglycemic patients with type 2 diabetes with the isotope dilution technique to measure hepatic glucose production (7).

Using the frequently sampled intravenous glucose tolerance test and the minimal model approach, we also reported such acute favorable effect of metformin in a population similar to that of the DPP, i.e., in subjects with impaired glucose tolerance with a significant increase of the insulin sensitivity index after only $48 \mathrm{~h}$ metformin therapy $(+50 \% ; P=0.03)(8)$. That part of the overall effect seen in the DPP may be accounted for by a pure pharmacological effect of metformin that did not persist when the drug was stopped (1) is in agreement with those experimental results (6-8). Furthermore, the rather short washout period of only 1-2 weeks may be questionable. First, it did not allow a sufficiently high number of new cases of diabetes, which limited the statistical power of the analysis (5) - the $P$ value was 0.098 despite a difference of $49 \%$ in diabetes rates between placebo and metformin groups during the washout period. In addition, while the washout period was beyond the classically requested period of five times the plasma half-life of the drug (9), it did not completely exclude the persistence of any biological effect of metformin, which has a particularly complex cellular mechanism of action. Thus, in our opinion and in agreement with Buchanan (5), the question remains open as to whether the results of the DPP with metformin correspond to preventing, delaying, or partially masking effects of type 2 diabetes.

\section{References}

1. Diabetes Prevention Program Research Group: Effects of withdrawal from metformin on the development of diabetes in the Diabetes Prevention Program. Diabetes Care 26:977-980, 2003

\footnotetext{
2. Diabetes Prevention Program Research Group: Reduction in the incidence of type 2 diabetes with lifestyle intervention or metformin. N Engl ] Med 346:393-403, 2002

3. Chiasson JL, Josse RG, Gomis R, Hanefeld M, Karasik A, Laakso M, STOP-NIDDM Trial Research Group: Acarbose for prevention of type 2 diabetes mellitus: the STOP-NIDDM randomised trial. Lancet 359:2072-2077, 2002
}

4. Scheen AJ: Acarbose for type 2 diabetes prevention (Letter). Lancet 360: 1516, 2002

5. Buchanan TA: Prevention of type 2 diabetes: what is it really? Diabetes Care 26: 1306-1308, 2003

6. Widen EIM, Eriksson JG, Groop LC: Metformin normalizes nonoxidative glucose metabolism in insulin-resistant normoglycemic firstdegree relatives of patients with NIDDM. Diabetes 41:354-358, 1992 


\title{
Preventing, Delaying, or Masking Type 2 Diabetes With Metformin in the Diabetes Prevention Program?
}

\author{
Response to Scheen \\ THOMAS A. BUCHANAN, MD \\ From the Departments of Medicine, Obstetrics and Gynecology, and Physiology and Biophysics, Keck School of Medicine, University of \\ Southern California, Los Angeles, California
}

I agree with the fundamental point made by Dr. Scheen (1) in this issue of Diabetes Care. It is unclear whether the effect of metformin to reduce the incidence of diabetes during the Diabetes Prevention Program (DPP) (2) was true prevention or simply a masking of diabetes. The posttrial washout period was very short for testing that distinction. The data cited by Dr. Scheen regarding a very rapid effect of metformin to increase insulin sensitivity suggests that dissipation of any such effect in the DPP could have been rapid as well. Thus, acute effects of metformin on insulin sensitivity could have been gone 2 weeks after the drug was stopped. It seems unlikely that we will ever know for sure because direct measures of insulin sensitivity were not made in the DPP. Additionally, we will never know whether glucose levels had stabilized or were still rising 2 weeks after metformin was stopped. The Study to Prevent (STOP)-NIDDM trial (3) included a more convincing washout period of 3 months before retesting for diabetes. During the washout, the jump in new cases of diabetes in the former acarbose group (twice as many as in the former placebo group) was even more striking than the analogous jump in the former metformin group in the DPP (49\% more cases than in the placebo group). Whether this greater jump in the STOP-NIDDM trial reflects the more meaningful washout period used in that trial or a greater component of masking rather than prevention of diabetes compared with metformin can't be determined from the published data.

We have provided strong evidence from the Troglitazone in the Prevention of Diabetes (TRIPOD) study (4), conducted in Hispanic-American women, that lowering endogenous insulin requirements can slow or stop the loss of $\beta$-cell function, which leads to type 2 diabetes. If this effect occurs in other ethnic groups (still a big "if"), then any effect of metformin or acarbose to lower glucose levels or enhance insulin sensitivity could have had an analogous, albeit probably weaker, effect in the DPP and STOP-NIDDM trials. Given the known effects of metformin and acarbose on carbohydrate metabolism, I suspect that portions of the reductions in diabetes rates observed in the metformin arm of the DPP and in the STOP-NIDDM trial were due to $\beta$-cell protection. The posttrial washouts in the two studies demonstrate that some of the apparent protection from diabetes was simply masking caused by acute glucose-lowering effects of the drugs.

We will never know precisely how much prevention and how much masking of diabetes occurred in the DPP and the STOP-NIDDM trials. Nonetheless, discourse on this issue can be helpful. It can focus diabetes prevention research on the real disease of type 2 diabetes - progressive $\beta$-cell failure - rather than on the glucose thresholds that currently define diabetes. Those thresholds are clearly important for assessing the risk of microvascular and neuropathic complications of diabetes. However, they are not very useful for tracking the underlying $\beta$-cell disease. That disease progresses for a long time before patients cross the glucose threshold to diabetes, and it continues after they have crossed that threshold. When the discussion turns to prevention (or even to initial treatment) of type 2 diabetes, the goal should become stability of glucose levels and $\beta$-cell function. 


\author{
References \\ 1. Scheen AJ: Preventing, delaying, or masking type 2 diabetes with metformin in the Diabetes Prevention Program? (Letter). Diabetes Care \\ 26:2701, 2003.
}

2. Diabetes Prevention Program Research Group: Effects of withdrawal from metformin on the development of diabetes in the Diabetes Prevention Program. Diabetes Care 26:977-980, 2003

3. Chaisson JL, Josse RG, Gomis R, Hanefeld M, Karasik A, Laakso M: Acarbose for prevention of type 2 diabetes: the STOP-NIDDM randomised trial. Lancet 359: 2072-2077, 2002

4. Buchanan TA, Xiang AH, Peters RK, Kjos SL, Marroquin A, Goico J, Ochoa C, Tan S, Berkowitz K, Hodis HN, Azen SP: Preservation of pancreatic $\beta$-cell function and prevention of type 2 diabetes by pharmacological treatment of insulin resistance in high-risk Hispanic women. Diabetes 51: 2796-2803, 2002

\title{
Response to Scheen and Buchanan
}

\author{
MARK E. MOLITCH, MD
}

From the Diabetes Prevention Program Coordinating Center, Biostatistics Center, George Washington University, Rockville, Maryland.

We appreciate the comments of Drs. Scheen (1) and Buchanan (2) regarding our article (3), which described the effects of withdrawal of metformin on diabetes status in the Diabetes Prevention Program (DPP). As Dr. Scheen comments, and as we pointed out in our article, clearly part of the effect of metformin was due to an acute pharmacological effect of metformin, but the great majority of the effect persisted beyond this period of washout. As he notes, the washout period of 1-2 weeks was indeed beyond the usual required period of five times the plasma half-life of the drug. The duration and nature of metformin's effects beyond that time frame are not well established. However, there are no data to support Dr. Scheen's conjecture that waiting for an additional time would "allow a sufficiently high number of new cases of diabetes," which he feels limited the statistical power of the analyses. The data are what they are. Within the DPP, the investigators felt that it would not be ethical to withhold such an efficacious treatment for a prolonged period of time to answer these questions. Furthermore, pharmacological treatment of many other chronic diseases, such as hypertension and dyslipidemia, is generally given continuously. We don't expect drugs treating these conditions to work after they are discontinued. Similarly, we shouldn't expect drug treatment of impaired glucose regulation and other risk factors for type 2 diabetes to be effective after the drugs are discontinued.

We agree that whether drug therapy with metformin, acarbose, troglitazone, estrogens, ACE inhibitors, or angiotensin II receptor blockers truly prevent diabetes or delay its onset cannot be determined completely by the existing studies. However, delaying the onset of diabetes is clearly an important goal with major individual and public health implications. From a practical standpoint, there is little difference between delay and prevention of a disease such as type 2 diabetes with variable and late onset. If an intervention could delay the onset of diabetes long enough such that a person ultimately dies without developing the disease, delay would be equivalent to prevention. Unfortunately, neither of the DPP interventions (4) was totally effective, i.e., neither reduced the diabetes incidence rate to zero. Thus they produced only partial delay or prevention. Even partial effectiveness in this regard, however, is beneficial and is cause for optimism that more effective interventions can be developed.

\section{References}

1. Scheen AJ: Preventing, delaying, or masking type 2 diabetes with metformin in the Diabetes Prevention Program? (Letter) Diabetes Care 26:2701, 2003

\section{Buchanan TA: Prevention of type 2 diabetes: what is it really? Diabetes Care 26: 1306-1308, 2003}


Published in: Diabetes Care (2003), vol. 26, iss. 9, pp. 2701; author reply 2701-3

Status: Postprint (Author's version)

4. The Diabetes Prevention Program Research Group: Reduction in the incidence of type 2 diabetes with lifestyle intervention or metformin. N Engl J Med 346: 393-403, 2002. 